\title{
A PRÁTICA CLÍNICA BASEADA EM EVIDÊNCIAS. PARTE I - QUESTÕES CLÍNICAS BEM CONSTRUÍDAS
}

\author{
Moacyr Roberto Cuce Nobre, Wanderley Marques Bernardo, fabio Biscegl Jatene*
}

Trabalho realizado na Associação Médica Brasileira, São Paulo, SP

RESUMO - Na prática diária as decisões tomadas para resolver 0 problema do paciente são usualmente baseadas na aplicação consciente da informação avaliável por regras explicitamente definidas. A prática clínica baseada em evidências leva em consideração o reconhecimento dos conhecimentos explícitos e tácitos, entendendo que é impossivel tornar explícito todos os aspectos da competência profissional. A dúvida passa a fazer parte do processo de decisão, inicialmente na identificação dos componentes inconscientes envolvidos, e em seguida na análise do conhecimento explícito utilizado nesse processo. Ao elaborarmos uma questão clínica estruturada e que possa ser respondida, devemos lembrar que essa dúvida pode estar relacionada a aspectos básicos e de definição da doença ou relacionada ao atendimento do paciente, como em diagnóstico, terapêutica ou prognóstico. Ao longo de nossa vida médica, ambos os tipos de questões estão presentes, variando na proporção em que nossa experiência aumenta à medida do tempo de prática clínica. 0 processo de encontrar resposta apropriada à dúvida surgida no atendimento depende da forma como estruturamos as partes deste processo. A forma preconizada é conhecida pela sigla PICO. formada por $\mathbf{P}$ de paciente ou população, I de intervenção ou indicador, C de comparação ou controle e $O$ de "outcome", que na língua inglesa significa desfecho clínico, resultado, ou por fim, a resposta que se espera encontrar nas fontes de informação científica. Esta é a primeira condição básica para que a nossa busca possa ser bem sucedida, a segunda é encontrar as palavras-chaves que melhor descrevem cada uma destas quatro características da questão. Sem estes cuidados as pesquisas em bases de dados informatizadas costuma resultar em ausência de informação ou em quantidade muito grande de informação que não está relacionada com o nosso interesse.

UnITERMOS: Medicina baseada em evidência. Decisão clínica. Atendimento ao paciente. Relação médico-paciente. Ciência da informação. "Outcome".

\section{INTRODUÇÃO}

Este artigo é o primeiro de três artigos de atualização sobre a prática clínica baseada em evidências. $O$ primeiro aborda a dúvida que surge no atendimento do paciente, e como ela pode ser transformada em uma questão clínica bem estruturada, que facilite a busca em fontes de informação. Os demais artigos enfocam como achar trabalhos científicos em revistas eletrônicas, como recuperar informações em bases de dados informatizadas, como avaliar criticamente a informação obtida e finalmente como aplicá-la no atendimento do paciente.

\section{A dúvida no atendimento ao paciente}

Na prática diária as decisões tomadas para resolver o problema do paciente são usualmente baseadas na aplicação consciente da informação avaliável por regras explicitamente definidas. Esta forma de conhecimento explícito

* Correspondência: Rua São Carlos do Pinhal, 324 CEP:01333-903 - São Paulo - SP pode ser quantificada, modelada, prontamente comunicada e facilmente transposta para diretrizes de conduta clínica baseadas em evidência.

No entanto, uma grande quantidade de conhecimento tácito, experiência, valores e habilidades constituem um tipo diferente de evidência, a qual tem uma forte influência na tomada de decisão. Enquanto os elementos explícitos são ensinados formalmente, os tácitos são adquiridos durante a observação e a prática'.

Toda informação compreendida, independentemente da sua veracidade, costuma ser aplicada na prática clínica. Aquelas que são explícitas podem ser criticamente avaliadas pela medicina baseada em evidências, no entanto, esta metodologia não é suficiente para descrever e incluir o processo tácito do julgamento clínico.

No processo tácito, fatores relacionados ao médico, como emoções, vícios de observação, percepção de prejuízos, aversão ao risco, tolerância quanto à incerteza e relacionamento pessoal com o paciente também influenciam, em menor ou maior grau, o julgamento clíni$\mathrm{co}$, muitas vezes de forma inconsciente.

A prática clínica baseada em evidências leva em consideração o reconhecimento dos conhecimentos explícitos e tácitos, entendendo que é impossível tornar explícito todos os aspectos da competência profissional. Adúvida passa a fazer parte do processo de decisão, inicialmente na identificação dos componentes inconscientes envolvidos, eem seguida na análise do conhecimento explícito utilizado nesse processo.

Em reuniões de trabalho do Projeto Diretrizes da Associação Médica Brasileira e Conselho Federal de Mediciana, discutiu-se as dificuldades do médico admitir e reconhecer as suas próprias dúvidas no atendimento ao paciente. Os principais elementos envolvidos como fatores limitantes na admissão da dúvida foram identificados como:

I. Medo de se expor - sem o autoconhecimento e sem o conhecimento do paciente, 0 profissional evita expor as suas limitações de conhecimento da doença, temendo a reação ou rejeição do paciente quanto a sua conduta; 
2. Falta de experiência - a experiência envolve o processo necessário principalmente em situações complexas de decisão, onde a urgência é o fator limitante. Nestas situações o médico age de modo sobretudo tácito e os conhecimentos explícitos adquiridos muitas vezes são pouco utilizados. A dúvida não pode ser reconhecida abordando-se apenas as evidências científicas;

3. Arrogância e prepotência - para se reconhecer a existência da dúvida é necessário o entendimento de que não se sabe tudo, e que é possível compartilhar a responsabilidade pela conduta com o paciente;

4. Não escutar, nem se colocar ao lado do paciente - o princípio de que a decisão depende essencialmente do conhecimento explícito adquirido, e que portanto independe do momento e em quem o conhecimento é aplicado, subestima a influência dos componentes tácitos, dificultando a admissão da própria dúvida;

5. Formação acrítica - o modelo de aquisição do conhecimento, através da via de mão única, "bancária" sem o devido tempo e estímulo para a reflexão, impede e desestimula a existência da dúvida, apesar das observações de Paulo Freire ${ }^{2}$, que data do final dos anos sessenta: "na prática bancária da educação, antidialógica por essência, por isto, não comunicativa, o educador deposita no educando o conteúdo programático da educação, que ele mesmo elabora ou elaboram para ele, ao contrário da prática problematizadora, dialógica por excelência, este conteúdo, nunca é depositado, se organiza e se constitui na visão do mundo dos educandos, em que se encontram seus temas geradores. Por isso mesmo, ninguém educa ninguém, como tampouco ninguém se educa a si mesmo, os homens se educam em comunhão, mediatizados pelo mundo".

O ideal da prática clínica baseada em evidências inclui uma prática reflexiva e cuidadosa, onde além da identificação da dúvida, medidas são tomadas momento a momento, com o objetivo de corrigir distorções e desvios de rumo, durante 0 processo de decisão médica. Então, como deve ser a prática clínica reflexiva, baseada em evidências? Segundo ainda as reuniões de trabalho do Projeto Diretrizes, alguns degraus devem ser percorridos, numa ordem que pode variar:
I. Aceitar a dúvida, procurando a resposta na melhor evidência, e após aplicar na prática, avaliar o desfecho;

2. Procurar o controle e a monitoração consciente dos fatores emocionais envolvidos;

3. Ouvir atentamente o paciente, procurando não o interromper, permitindo que participe da tomada de decisão;

4. Não sofrer pelo, mas com o paciente, na tomada de decisão;

5. Ampliar as evidências, levando em consideração o conhecimento tácito adquirido, de acordo com a visão periférica';

6. Trazer o conhecimento epidemiológico para $o$ atendimento individual;

7. Estabelecer prioridades, sistematizando e hierarquizando o processo de decisão.

\section{Dúvidas de condutas que se originam no cenário clínico}

Muitos dos procedimentos que tomamos no dia-a-dia são feitos de forma tácita, sem nenhum questionamento crítico. Imagine um cenário no qual o paciente se queixa de desconforto devido a sensação de calor excessivo, acompanhada de calafrios, e temperatura maior do $39^{\circ}$. C. Tomando alguns cuidados para não atrapalhar o processo diagnóstico, 0 profissional deve empregar medidas anti-térmicas, que não apresentem contra-indicação para o paciente em questão. A totalidade dos profissionais bem formados poderiam seguir esta conduta de forma automática, com a certeza de que estariam fazendo o melhor para o paciente.

No entanto, outras vezes o processo de tomar decisões é mais complexo, e temos dúvidas sobre quais os procedimentos mais indicados. Quando somos recém-formados costumamos atribuir esta situação à insegurança própria desta fase da vida, que deve desaparecer a medida que acumulamos experiência na prática de cuidados à saúde. Mais tarde percebemos que parte destas dúvidas permanecem, apesar da experiência acumulada. Não nos convencemos que parte do que é habitualmente recomendado pelos livros de texto se fundamenta em empirismo cientificamente elaborado. Em outras palavras, nos damos conta da necessidade de nos permitir questionar a conduta, para em seguida procurar evidências que a fundamentem.

A maioria de nós tivemos a formação básica e universitária clássica, na qual o senso crítico é pouco desenvolvido, o princípio do aprendizado é acumulativo, com grande quantidade de informações estanques, voltadas para o contexto da doença, ou pior ainda, de seus marcadores biológicos. Prevalecem as condutas que são tomadas a partir de rótulos que reduzem o ser humano à condição de simplesmente diabéticos, fibromiálgicos, hipercolesterolêmicos, HIV positivos, ou portadores de mutação genética BRCAI/BRCA2. As pessoas não são observadas dentro de seu contexto de vida. Oraciocínio para tomada de decisões costuma ser feito em base explanatória, e não empírica, em função de mecanismos fisiopatológicos e alterações estruturais, e não em razões probabilísticas e medida de associação epidemiológica. A medicina é muito mais do que solicitar exames que empregam tecnologia sofisticada de biologia molecular ou de diagnóstico por imagem ${ }^{3}$, por mais que queiram o interesse do mercado ou a clientela que segue os preceitos da moda.

É preciso uma mudança radical deste paradigma, é necessário que o profissional passe a avaliar as outras características de sua clientela que não fazem parte do contexto biológico. Essas pessoas podem ser consideradas hipertensas, diabéticas e coronariopatas, mas além disso, elas tem nome, trabalham, se divertem, são pais, irmãs e têm amigos. Em benefício da qualidade de vida delas, e não de um marcador biológico, o profissioanal de saúde deve dirigir os seus esforços.

Éesta mudança de paradigma que a prática clínica baseada em evidências tem perseguido desde que foi lançada em 1992 como o nome de Evidence-Based Medicine ${ }^{4}$. Neste artigo é apresentado o cenário clínico no qual um homem de 48 anos é avaliado pelo residente, por apresentar uma primeira crise convulsiva, não acompanhada por nenhuma outra queixa de saúde, nem antecedente traumático ou de ingestão de bebidas alcoólicas associado ao episódio, tomografia de crânio normal e eletroencefalograma com achados inespecíficos. O paciente apresenta-se extremamente preocupado com a possibilidade da crise epilética recorrer. Como o residente deve proceder?

Novelho paradigma, oresidenteéinstruído pelo preceptor, que por sua vez foi instruído pelo médico assistente, de que deve informar ao paciente sobre a alta possibilidade de recorrência da crise, possibilidade essa que não 
pode ser estimada em números. O paciente sai com uma sensação de total insegurança. No novo paradigma, após perguntar a si próprio sobre o prognóstico deste tipo de paciente, 0 residente se dá conta que não sabe. Ele então se dirige à biblioteca, como poderia acionar a sua agenda de mão conectada à Internet, e faz uma busca no PubMed, utilizando os termos "epilepsy", "prognosis", "recurrence" e como resultado recupera 25 artigos relevantes na base de dados. Lendo os títulos, seleciona um dos artigos relevantes, por se apropriar exatamente ao paciente em questão. Faz então uma avaliação crítica da validade do estudo prognóstico selecionado e conclui pela boa qualidade das informações contidas nele. Este procedimento custa aproximadamente $R \$ 2,50$ e leva cerca de meia hora para ser feito.

Volta ao paciente e dáa informação de que o risco de recorrência em I ano está entre 43 e $51 \%$, após um período assintomático de 18 meses o risco anual cai para 20\%. O paciente sai orientado no sentido de tomar a medicação, fazer o seguimento habitual com o clínico geral $e$ avaliar a possibilidade de suspender 0 anticonvulsivante se permanecer assintomático por 18 meses. Diferentemente do paradigma anterior, dentro da proposta da medicina baseada em evidências o paciente recebe informação mais precisa, que lhe propicia participar da tomada de decisão clínica e Ihe favorece o controle da ansiedade própria das condições desconhecidas, com conseqüente alivio da carga emocional decorrente da doença.

Se neste cenário a dúvida é explicita ejáse apresenta plenamente elaborada pelo próprio paciente, na prática cotidiana nem sempre se dá desta forma. As questões clínicas que precisam ser respondidas no atendimento ao paciente são várias, de natureza tácita e explícita, se relacionam entre si em diferentes graus de complexidade, difíceis de serem identificadas pelos profissionais não acostumados à este processo, mesmo por aqueles que admitemaexistência da dúvidasem maior dificuldade.

Como exemplo hipotético, consideremos uma senhora de 56 anos, moderadamente obesa, com diagnóstico de diabetes tipo II não complicada. A sua glicemia encontra-se bem controlada com metformina, no entanto a pressão arterial encontra-se pouco elevada, com valor médio de $158 / 94 \mathrm{mmHg}$ nas últimas três consultas. Orientações repetidas nos últi- mos quatro anos para que reduzisse o peso corpóreo não foram bem sucedidas. A paciente resiste à sugestão de utilizar mais medicamentos, preferindo "remédios naturais". 0 profissional de saúde está disposto a convencê-la a usar um antihipertensivo e para tanto gostaria de ter mais informações sobre os reais benefícios desta conduta, se ela traria maiores vantagens do que danos ao paciente diabético com as características do caso em questão. Como elaborar bem esta questão, de forma a facilitar a obtenção de informações?

\section{Questões clínicas (foreground questions) e dúvidas básicas (background questions)}

Ao elaborarmos uma questão clínica estruturada e que possa ser respondida, devemos lembrar que essa dúvida pode estar relacionada a aspectos básicos e de definição da doença ou relacionada ao manuseio do paciente, como em diagnóstico, terapêutica ou prognóstico.

Então, poderíamos dividir as questões clínicas em dois subgrupos principais: questões básicas (background) e questões clínicas (foreground):

I. As perguntas estruturadas advindas de dúvidas básicas possuem duas características principais: a) um pronome ou advérbio interrogativo associado a um verbo, determinando a raiz da questão (por quê, como, quando, onde, quem, o quê, qual); b) uma doença ou um aspecto desta. São questões relacionadas à etiologia, etiopatogenia, fisiopatologia, epidemiologia e não envolvem o manuseio dos pacientes. Então, por exemplo: Qual a causa da pneumonia comunitária? Por quê ocorre 0 derrame pleural na pneumonia? Qual a sua frequêencia populacional.

2. As questões clínicas enfocam o conhecimento a respeito do cuidado aos pacientes com uma determinada doença, possuindo como componentes principais: a) o paciente ou problema de interesse; b) a intervenção principal que pode incluir uma exposição, um método diagnóstico, um fator prognóstico, um tratamento, ou ambos; c) uma intervenção de comparação, quando cabível; d) os desfechos clínicos de interesse. São questões que abordam claramente aspectos de diagnóstico, tratamento e prognóstico aplicáveis a um paciente com uma determinada doença. Então, por exemplo: Os achados clínicos são suficientes para fazer diagnóstico de pneumonia, ou a radiografia de tórax é sempre necessária? O prognóstico de um paciente tratado, com pneumonia comunitária, em ambulatório é pior do que aquele em nível hospitalar?

Ao longo de nossa vida médica, ambos os tipos de questões estão presentes, variando na proporção em que nossa experiência aumenta à medida do tempo de prática clínica. Assim, no início da prática médica, há um componente maior de questões básicas, o qual tende a diminuir com o passar do tempo, dando lugar a um componente cada vez maior de questões clínicas. No entanto, é importante entendermos que, nunca o médico é tão inexperiente, que não possa adquirir o conhecimento clínico, como também nunca é tão experiente, que não tenha dúvidas básicas ${ }^{5}$.

A prática clínica diária, sobretudo baseada em evidências, exige que usemos grande quantidade de conhecimento, tanto básico como clínico. As questões então, surgem de maneira híbrida, estando centradas no cuidado aos pacientes, e num cenário comum, que envolve achados clínicos, etiologia, diagnóstico diferencial, métodos diagnósticos, fatores prognósticos, métodos terapêuticos, experiência e opinião do paciente e aprimoramento pessoal.

As questões clínicas nos alertam sobre possíveis benefícios e danos decorrentes $\mathrm{da}$ tomada de decisão frente ao paciente ou dos medicamentos, têm em comum o estudo das manifestações clínicas, dos sintomas, ou em última análise, o bem estar do paciente.

As questões básicas funcionam como pré-requisito à comprensão das questões clínicas, mas não as substituem na tomada de decisão. Aqui encontramos um dos principais equívocos na formação médica, habitualmente alimentados pelos interesses de mercado criados pelas indústrias de equipamentos diagnósticos e produtos farmacêuticos. Saber como funciona um método de aplicação diagnóstica, como por exemplo o comportamento da $\mathrm{HbAIC}$, também conhecida como hemoglobina glicada, em estados hiperglicêmicos é essencial ao patologista clínico, como também pode auxiliar o clínico a entender a eventual valia nos cuidados ao paciente. No entanto, o valor clínico deste conheci- 
NOBRE MRC ET AL.

Tabela - PICO

FORMULAR UMA QUESTÃO BEM CONSTRUÍDA, QUE PODE SER RESPONDIDA MAIS FACILMENTE

Questãopor Extenso:

Paciente ou População:

Intervenção oulndicador:

Comparação ou Controle:

Outcomeou Desfecho:

\section{PROCURARA(S) MELHOR(ES) EVIDÊNCIA(S)}

Estratégia de Busca:

DESCRITORBÁSICO

SINÔNIMO I

SINÔNIMO 2

$\mathbf{P}$ (

OR

OR

\begin{tabular}{llll}
\hline$I($ & OR & OR & )AND \\
\hline$C($ & OR & OR & )AND \\
\hline$O($ & OR & OR & AND
\end{tabular}

Considere truncar as palavras com “*”, por exemplochild* em vez de children

\begin{tabular}{|l|c|c|c|c|}
\hline \multicolumn{5}{|c|}{ EVIDÊNCIASENCONTRADAS } \\
\hline Fontesprimárias & $n^{\circ}$ & & Fontessecundárias \\
\hline & & & & \\
\hline & & & \\
\hline
\end{tabular}

mento só se estabelece a partir da sua observação empírica decorrente do seguimento de pacientes pelo tempo necessário para o surgimento de complicações clínicas do diabete.

Em outras palavras, por mais que o conhecimento molecular do processo de glicolização proteica demonstre que a quantidade de hemoglobina glicada no sangue circulante estime a persistência do estado hiperglicêmico por período prolongado de tempo, ao contrário da medida da glicemia que mede unicamente o momento da coleta da amostra, este conhecimento não é suficiente para garantir o eventual benefício prognóstico que poderia advir do controle medicamentoso dos nivel de $\mathrm{HbAIC}$. Ao contrário, evidências relatadas pelo estudo conhecido por UKPDS mostra que o rígido controle da pressão arterial, e não da glicemia, previne melhor a mortalidade precoce da doença.

O estudo UKPDS - United Kingdom Prospective Diabetes Study, que fornece evidências de muita boa qualidade metodológica, mostrou que o rígido controle da glicemia reduz as complicações microvasculares, como a nefropatia e retinopatia, mas praticamente não tem efeito sobre os desfechos macrovasculares. No entanto, cerca de $80 \%$ dos pacientes dia- béticos irão desenvolver ou morrer precocemente por complicações macrovasculares do tipo infarto do miocárdio ou acidente vascular cerebral ${ }^{6}$.

Elaborando questões clínicas bem construídas e desenhando a estratégia de busca

O processo de encontrar resposta apropriada à dúvida surgida no atendimento depende da forma como estruturamos as partes deste processo. A forma preconizada é conhecida pela sigla $\mathrm{PICO}^{7}$. formada por $\mathrm{P}$ de paciente ou população, I de intervenção ou indicador, $\mathrm{C}$ de comparação ou controle e $\mathrm{O}$ de 
"outcome", que na língua inglesa significa desfecho clínico, resultado, ou por fim, a resposta que se espera encontrar nas fontes de informação científica. Esta é a primeira condição básica para que a nossa busca possa ser bem sucedida, a segunda é encontrar as palavras-chaves que melhor descrevem cada uma destas quatro caracteríticas da questão. Sem estes cuidados as pesquisas em bases de dados informatizadas costuma resultar em ausência de informação ou em quantidade muito grande de informação que não está relacionada como nosso interesse.

No caso exemplificado acima o "P" corresponde a pessoas idosas do sexo feminino com diabetes tipo Il e hipertensão arterial não complicadas, o "I" corresponde ao tratamento antihipertensivo, o "C" não tratar a hipertensão ea informação "O" que se pretende recuperar enquanto "outcome" ou desfecho clínico corresponde ao benefício para a saúde da paciente em questão. Se considerarmos que a principal causa de morte destes pacientes é a doença cardiovascular poderíamos reformulara estratégia de busca mantendo o "P", substituindo "l" por controle medicamentoso da hipertensão e o "C" por controlemedicamentoso da glicemia. A questão então seria: $O$ controle medicamentoso dahipertensãoé mais eficiente no aumento da sobrevida de pacientes idosas com hipertensão arterial e diabetes tipo II não complicadas, do que o controle medicamentoso da glicemia? Preferindo a primeira ou a segunda pergunta estruturada já temos as partes das perguntas que podemos identificar como descritores, ou palavras-chaves.

\section{SUMMARY}

EVIDENCE BASED Clinical PRACTICE. Part I - well structured clinical QUESTIONS

Clinical decisions in daily practice, to resolve patient's problem, are usually based at the conscious use of the avaliable information, through explicit determined rules. Evidence based clinical practice recognize the explicit and tacit knowledge, understanding that it is impossible all the aspects of professional competence become explicit. The doubt becomes part of the decision process, identifying initialy the inconcious component envolved and after the explicit knowledge used. When we make a stuctured clinical question with a possible answer, it is necessary to remember that the doubt can be relationned to basics and of definition aspects of the disease or relationned to the patient's mananger, like diagnose, treatment and prognose. Along our medical life, both types of question are present, with proportional change as the experience increase along the clinical practice. The process to find an appropriate answer to the doubt, came out at patient's care, depends on how the parts of this process will be structured. The recommended form is known by PICO abreviature, that means: $P$ : patient or population, I: intervention or indicator, $\mathrm{C}$ : comparison or controle and $\mathrm{O}$ : outcome, or the answer expected found at the cientific information bases. This is the first basic need to a successfull search, and the second need is to find the key words that better describe each of the four components of the questions. Without this caution, the search at compute databases results in absence of information or in a lot of information that it is not related to our interest. [Rev Assoc Med Bras 2003; 49(4): 445-9]

KEY WORDS: Evidence based medicine.Clinical pathway. Patient centered care. Physician patient relation. Information science. Outcome.

\section{REFERÊNCIAS}

I. Epstein RM. Mindful practice. JAMA. 1999; 282(9):833-9.

2. Freire P. Pedagogia do oprimido. $5^{a}$ ed. Rio de Janeiro: Paz e Terra; 1978.

3. VandenbrouckeJP. Evidence-based medicine and "medecine d'observation". J Clin Epidemiol I 996;49( I 2): I 335-8.

4. Evidence-Based Medicine Working Group. Evidence-based medicine. A new approach to teaching the practice of medicine. JAMA. 1992; 268(I7):2420-5.

5. Sackett DL, Straus S, Richardson S, Rosenberg W, Haynes RB. Evidence-based medicine: how to practice and teach EBM. $2^{\text {a }}$ ed. Londres: Churchill Livingstone; 2000. p. 17.

6. Hunt DL, Jaeschke R, McKibbon KA. Users' guides to the medical literature: $X X I$. Using electronic health information resources in evidence-based practice. Evidence-Based Medicine Working Group. JAMA. 2000; 283(14): 1875-9.

7. Richardson WS. Ask, and ye shall retrieve. Evidence-Based Medicine. 1998; 3: 100-I.

Artigo recebido: 22/09/2003

Aceito para publicação: 29/09/2003 